\title{
OSCILLATING-CURRENT CIRCUITS BY THE METHOD OF GENERALIZED ANGULAR VELOCITIES
}

\author{
BY V. BUSH
}

\section{ABSTRACT OF PAPER}

The oscillating-current circuit differs from the alternatingcurrent circuit in that the sinusoids involved are damped.

In the same way that alternating-current theoremsareobtained by generalizing direct-current theorems, it is possible to obtain oscillating-current theorems by still further generalization. This involves a generalized angular velocity and a generalized impedance which are complex quantities. Kirchoff's law may be thus generalized; and yields a simple method of determining the generalized angular velocities, and thus the frequencies and decrements, of free oscillation.

Since, in an oscillating-current circuit, there is no impressed voltage, the initial voltage present in the circuit must be used in determining the amplitudes of oscillation. This results in the introduction of a "threshold impedance" for use in determining the amplitudes, which is obtained by a single differentiation from the generalized impedance. Further direct-current rules may also be readily generalized for convenient use in the solution of oscillating-current circuits.

A simple example is given to show the method of procedure. The discharge of a leaky condenser through a reactor furnishes a second example which better illustrates the convenience of the method for numerical computations. As a third example the oscillation of a three-section artificial cable under particular terminal conditions is chosen. The algebraical portion of this example is given in an appendix.

A list of symbols used will be found at the end of the paper.

\section{INTRODUCTORY}

IN SOLVING alternating-current networks it is convenient to make use of the pure imaginary, $j$. A sinusoidally varying quantity is represented by a revolving plane vector, and this in turn by the expression:

$$
A \epsilon^{j \omega t}
$$

$A$ is the amplitude, and $\omega$ the "angular velocity" of the harmonic quantity or of the vector which represents it. When such expressions are combined according to the laws of algebra, true physical results are obtained because of the fact that harmon- 
ically varying quantities combine in accordance with the same laws as does this easily handled mathematical expression.

The use of this symbolism much simplifies the solution of such circuits; and much of this simplification results from the translation of direct-current rules into alternating-current rules by a proper exchange of terms. Thus Ohm's law is retained in its simplicity for the alternating-current case by the substitution of impedance for resistance; and the impedance of a circuit is defined in such a manner that the law will hold.

In oscillating-current circuits we consider the subsidence of a system from one state to another in the absence of impressed forces. The quantities involved vary in accordance with damped sinusoids. They may be represented by revolving plane vectors, which shorten exponentially as they revolve. Such a vector may be represented by the expression:

$$
A \epsilon^{n \iota}
$$

where $n$ is a complex quantity of the form:

$$
n \equiv-\alpha+j \omega
$$

If we rewrite the expression in the form:

$$
A \epsilon^{-a t} \epsilon^{j w t}
$$

it is seen to consist of the term of the alternating-current case multiplied by a damping factor. In such an expression the complex quantity $n$ may be conveniently called a generalized angular velocity; and we may form from it generalized impedances, generalized admittances etc. in a manner exactly analagous to the alternating-current case.

\section{The Frequencies and Decrements of Free Oscillation}

Kirchoff's law, that the sum of the voltages around a closed path in a direct-current circuit is zero, is translated for use in alternating-current circuits by substituting the term impedance for resistance.

In similar manner it may be extended for use in oscillatingcurrent circuits by putting in instead the term, generalized impedance, Since there are in this case no impressed voltages to be considered, the law may be more conveniently expressed by stating that the generalized impedance of a closed circuit to free oscillations is zero. 
In the direct-current case the law furnishes an equation for determining an unknown constant. In the alternating-current case it may be used also to determine the frequency if everything else is known. In the oscillating-current case it may be used to determine the unknown generalized angular velocities of oscillation. This proposition was used by Rayleigh and Heaviside; and has been presented in more explicit form for convenient use by Eccles, Campbell, and Kennelly. $\dagger$

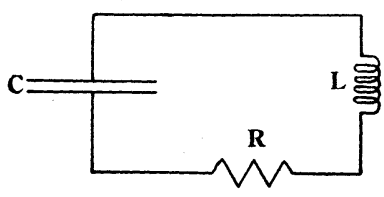

Fig. 1-Simple Series Circuit

One example will be sufficient to show the convenience of the law for practical use. Consider the series circuit of Fig. 1; a condenser of capacitance $C$, discharging through a reactor of inductance $L$ and resistance $R$.

If we let $n$ be the unknown generalized angular velocity, and apply the law to this circuit, we obtain

$$
R+L n+\frac{1}{C n}=0
$$

From which, solving for $n$, there results:

$$
n=-\frac{R}{2 L} \pm j \sqrt{\frac{1}{L C}-\left(\frac{R}{2 L}\right)^{2}} .
$$

Assuming for convenience the quantity under the radical positive, the angular velocity of free oscillation of this circuit is thus:

$$
\sqrt{\frac{1}{L C}-\left(\frac{R}{2 L}\right)^{2}}
$$

or the frequency of oscillation is

$$
\frac{1}{2 \pi} \sqrt{\frac{1}{L C}-\left(\frac{R}{2 L}\right)^{2}}
$$

$†$ Rayleigh, Theory of Sound.

Heaviside, Electromagnetic Theory, Vol. 2.

Eccles, Electrician, 1915. Phys. Soc. Proc. 24, 1912.

Campbell, Trans. A. I. E. E., 1911. Vol. XXX, Part II, p. 873.

Kennelly, Proc. Institute Radio Engineers, 1915. 
and the circuit has a decrement expressed by

$$
\frac{R}{2 L}
$$

This familiar result, in which there may be three cases according to the sign of the quantity under the radical, is thus arrived at simply by the solution of an algebraic equation, without the necessity of employing the calculus.

\section{The Amplitudes of Free Oscillation}

If we attempt to translate Ohm's law in analogous manner, in order to arrive at the amplitudes of oscillation, a difficulty arises because of the fact that there is no impressed voltage present in an oscillating-current circuit. The impressed voltage, and the generalized impedance, are both zero; and their quotient is indeterminate.

There is however always an initial voltage present in such a circuit due either to an initial charge on a condenser, or to an initial current through a resistor. We seek then a function of the constants of the circuit, the generalized impedance $Z$, and the free generalized angular velocities $n$ which shall give the amplitudes of oscillation when divided into the initial voltage.

Such a function will be found in the expression.

$$
n \frac{d Z}{d n}
$$

It may well be called on account of its peculiar properties the "threshold impedance" of the circuit.

.If a circuit, initially containing a voltage $E$, be allowed to oscillate freely, the initial amplitude of current oscillation will be.

$$
\frac{E}{n \frac{d Z}{d n}}
$$

and the current expression accordingly, the sum of terms of the form

$$
\frac{E}{n \frac{d Z}{d n}} \epsilon^{n \iota}
$$


where each term is formed from one of the values of $n$ resulting from the solution of the equation

$$
Z=0
$$

$Z$ is formed for the entire circuit, considering the element initially charged as the main branch.

The proof of this second law is neccesarily long; and may well be omitted in a paper of this character. The "threshold impedance" appears in papers by Heaviside.

When there is only a single frequency of oscillation present, as in the series circuit treated above, this second law is unnecessary; for the amplitude may be written down by inspection. In more involved networks, where several frequencies of oscillation exist simultaneously, the law furnishes a very convenient means of determining the amplitudes of the several terms in the solution.

\section{Solution of Circuits}

To completely solve an oscillating-current network, the method of generalized angular velocities thus requires the following steps:

1. Form the generalized impedance of the network, considering as the main branch the element initially charged.

2. Equate to zero, and solve for the generalized angular velocities of free oscillation: $n_{1} n_{2} n_{3} \ldots$.

3. Form the threshold impedance,

$$
T=n \frac{d Z}{d n}
$$

and insert the roots for $n$ found above to form a series of values of the threshold impedance: $T_{1} T_{2} T_{3} \ldots$.

4. Divide the initial voltage $E$ of the circuit by each of these values to form a corresponding set of amplitudes.

5 . Write the oscillating-current expression in the form

$$
i=\frac{E}{T_{1}} \epsilon^{n_{1} t}+\frac{E}{T_{2}} \epsilon^{n_{2} t}+\frac{E}{T_{3}} \epsilon^{n_{3} t}+. . .
$$

$\ddagger$ Heaviside, Electrical Papers, Vol. 2, p. 373., Electromagnetic Theory. Vol. 2, p. 135.

Since this paper was written, an excellent proof of the second law by Wagner has appeared in Archiv für Elektrotechnik, 1916, Band IV, 5/6 Heft. 
or more concisely,

$$
i=\sum \frac{E}{T} \epsilon^{n t}
$$

where the summation extends over the values of $n$ found from the equation,

$$
Z=0
$$

It will be noted that in the above the generalized angular velocities, generalized impedances, threshold impedances and generalized amplitudes are all complex quantities. Upon reducing the final expression to the usual trigonometric form by the use of the identity,

$$
\boldsymbol{\epsilon}^{j \omega t} \equiv \cos \omega t+j \sin \omega t
$$

the imaginary portions of the expression will of necessity cancel out, and leave a real trigonometric expression for the current.

Further rules for the examination of oscillating-current circuits may be readily obtained by generalization.

The current or voltage in a distant portion of the network may be found by combining the generalized impedances of the elements in the manner of simple resistances. Here as before the several terms of the current or voltage expressions are to be treated separately. An element has a generalized impedance corresponding to each free generalized angular velocity, to be used with the term of the current or voltage expression containing the same value of $n$.

When several stores of energy are simultaneously discharged, they may be treated separately and the results added.

Cases of oscillation under a suddenly applied steady electromotive force may be treated as the inverse of the discharge from the final state attained.

Oscillations under suddenly applied alternating voltages may be similarly treated; but the solution here rapidly becomes involved algebraically.

Examples of the Method. Three examples of the practical use of the method will be given. The first is very simple, to outline the procedure only. The second is of somewhat more complexity and is solved numerically. The third is intended to exemplify the method when several frequencies are present simultaneously. The inductively coupled circuit, which furnishes an excellent 
example for this purpose, is reserved because of its interest. for separate treatment. The method as used has been checked in numerous cases by the use of parallel solutions of the differential equation. Circuits such as that of example III have also been checked by the oscillograph and the artificial line at Harvard University.

I. Consider the circuit of Fig. 2: a condenser of capacitance $C$, initially charged to potential $E$, and discharging through simple resistance $R$.

The generalized impedance is,

$$
Z=R+\frac{1}{C n}
$$

ohms $\angle$

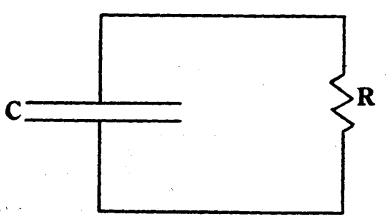

Fig. 2-Circuit of Example I

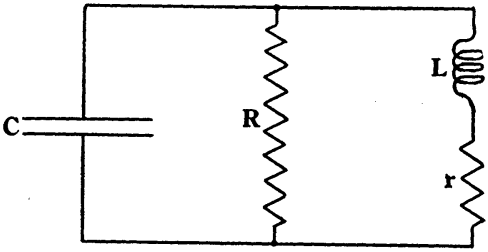

Fig. 3-Circuit of Example II

Equate to zero, solve for $n$, and there results,

$$
n=-\frac{1}{R C} \quad \text { hyp. rad./sec. }
$$

The threshold impedance is obtained from $Z$ by differentiation;

$$
T=n \frac{d Z}{d n}=-\frac{1}{C n}
$$

For the free value of $n$ found, this has the value,

$$
T=-\frac{1}{C\left(-\frac{1}{R C}\right)}=R
$$

The current in the circuit is thus,

$$
i=\frac{E}{T} \epsilon^{n t}=\frac{E}{R} \epsilon^{-\frac{t}{R C}}
$$


which may be checked by inspection and is the well known result.

II. As a better example, consider the network of Fig. 3; a condenser of capacitance $C$, initially charged to potential $E$, shunted by resistance $R$, and discharging through a coil of inductance $L$ and resistance $r$.

Consider the condenser as the main branch, and form the generalized impedance:

$$
\begin{aligned}
Z_{\mathrm{c}}=\frac{1}{C n} & +\frac{1}{\frac{1}{R}+\frac{1}{r+L n}} \\
& =\frac{R+r+L n+R r C n+R L C n^{2}}{C n(R+r+L n)} \text { ohms } \angle
\end{aligned}
$$

Equate to zero, and solve for $n$, and there results,

$$
n=-\left(\frac{r}{2 L}+\frac{1}{2 R C}\right) \pm j \sqrt{\frac{1}{L C}-\left(\frac{r}{2 L}+\frac{1}{2 R C}\right)^{2}} \text { hyp. }
$$

It may be noted in passing that if we had formed $Z$ with another branch, say $R$, as the main branch;

$$
\begin{aligned}
Z_{\mathrm{R}}=R+ & \frac{1}{C n+\frac{1}{r+L n}} \\
& =\frac{R+r+L n+R r C n+R L C n^{2}}{1+r C n+L C n^{2}} \quad \text { ohms } \angle
\end{aligned}
$$

the same roots for $n$ would have resulted upon equating to zero. Owing to the difference in the denominator, however, $Z_{\mathrm{c}}$ should be used in forming the threshold impedance.

Form the threshold impedance:

$$
\begin{aligned}
& T=n \frac{d Z_{\mathrm{C}}}{d n} \\
& =\frac{\left(R^{2} L C^{2}-L^{2} C\right) n^{2}-2 L C(R+r) n-C(R+r)^{2}}{C^{2} n(L n+R+r)^{2}} \text { ohms } \angle
\end{aligned}
$$


For the sake of brevity let us now introduce the numerical values of the constants:

$$
\begin{aligned}
& C=10^{-5} \text { farad } \\
& L=0.1 \text { henry. } \\
& r=10 \text { ohms. } \\
& R=100 \text { ohms } \\
& E=100 \text { volts. }
\end{aligned}
$$

Substitute these values in the expressions above, and we obtain

$$
\begin{aligned}
& n=-550.0 \pm j 835.2 \\
& T=+101.2 \pm j 153.8
\end{aligned}
$$

We may now write the expression for the oscillatory current in the condenser,

$$
\begin{aligned}
i_{\mathrm{c}}=\frac{100}{101.2-j 153.8} \epsilon^{(-550.0+j 835.2) t} & \\
& +\frac{100}{101.2+j 153.8} \epsilon^{(-550.0-j 835.2) t} \text { amperes } \angle
\end{aligned}
$$

Note that in this expression each threshold impedance is associated with the term containing the generalized angular velocity from which it was derived.

Reduce the expression to trigonometric form, and combine terms, and it becomes,

$$
i_{\mathrm{c}}=1.089 \epsilon^{-550.0 t} \sin (835.2 t+0.9882) \quad \text { amperes. }
$$

Suppose we now wish the current in the coil. The condenser current divides between the other two branches in the ratio of their generalized admittances.

This ratio is,

$$
\frac{R}{R+(r+L n)}
$$

numeric $\angle$

or numerically,

$$
0.550 \pm j 0.8352
$$

numeric $\angle$

Multiply the exponential expression for $i_{\mathrm{c}}$ by this ratio; paying due attention to signs, and the coil current results:

$$
\begin{aligned}
i_{\mathcal{L}}=(-j 0.5443) & \epsilon^{(-550.0+j 835.2)^{t}} \\
& +(j 0.5443) \epsilon^{(-550.0-j 835.2) t} \text { amperes } \angle
\end{aligned}
$$


and this may be reduced to,

$$
i_{\mathrm{L}}=1.0886 \epsilon^{-550.0 t} \sin 835.2 t \quad \text { amperes. }
$$

If we desire the voltage across the inductance $L$, multiply $i_{\mathrm{L}}$ by $L n$, or by,

$$
(55.0 \pm j 83.52)
$$

ohms $\angle$

and obtain on reducing,

$$
e_{\mathrm{L}}=108.9 \epsilon^{-550.0 t} \sin (835.2 t-0.9882)
$$

and so on.

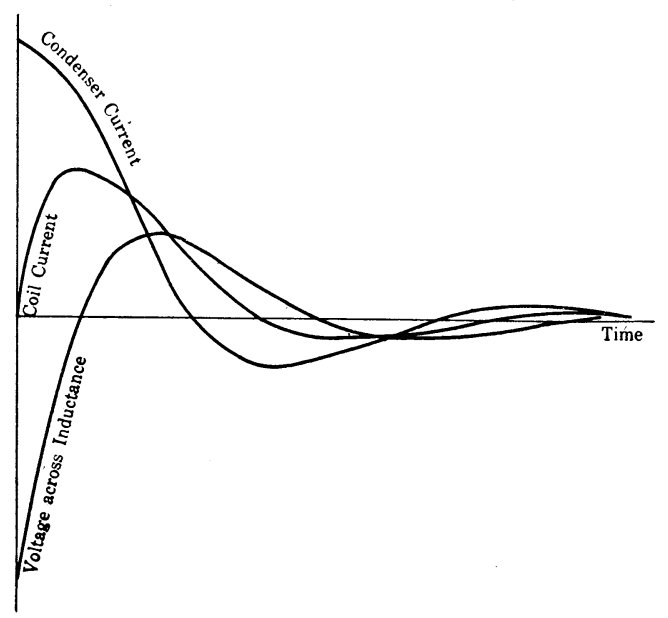

Fig. 4-Discharge of Leaky Condenser-Example II

These values are plotted in Fig. 4.

III. In the above example there are two conjugate roots for $n$, and hence a single frequency of free oscillation. The third example shows a case where more than one frequency is present. It is chosen from a series of solutions of the oscillations of the lumped artificial line under suddenly applied voltage. The three section artificial cable, grounded at one end, and suddenly connected at the other to a source of potential $E$, is chosen; as it illustrates the method with the least complexity of expression. The method proved adequate for the solution of the more general case with inductance present; and a series of solutions for various numbers of sections was used to derive a general expression for the lumped line and this type of transient. This expression, 
compares with that of a smooth line under similar conditions, furnished a criterion of the value of experimental results obtained on the lumped line for this type of transient. A lower limit for the number of sections could be set if such results were to apply to the smooth line case with allowable error.

If the total cable capacitance is $C$, and resistance $R$, the three section artificial cable is as shown in Fig. 5.

Putting for brevity,

$$
\begin{aligned}
& c=\frac{C}{3} \\
& r=\frac{R}{3}
\end{aligned}
$$

this circuit is equivalent to that of Fig. 6.

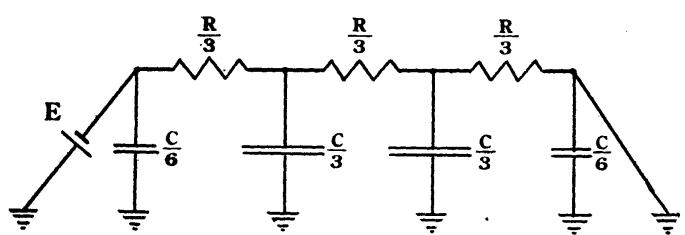

Fig. 5-Three Section Artificial

CABLE

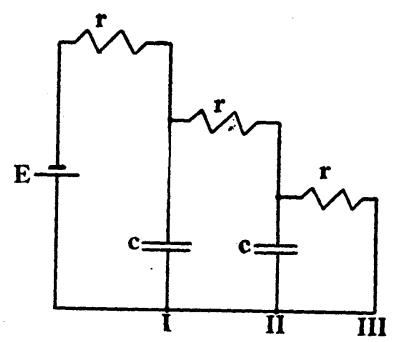

Fig. 6-Circuit of

EXAMPLE III

This is a case of charge, to be treated as the inverse of discharge from the final state attained. We are interested merely in the current in branch III.

In the steady state there is a charge in condenser I with terminal voltage: $\frac{2 E}{3}$, and in condenser II: $\frac{E}{3}$.

If we compute the discharge current of each of these condensers, by the method of the previous example, and find the proportional part of each which passes through branch III, we obtain respectively,

$$
\begin{aligned}
& i_{\mathrm{III}}^{\prime}=\frac{E}{3 r} \epsilon^{-\frac{T}{r c}}-\frac{E}{3 r} \epsilon^{-\frac{3 T}{r c}} \\
& i_{\mathrm{III}}^{\prime \prime}=\frac{E}{6 r} \epsilon^{-\frac{T}{r c}}+\frac{E}{6 r} \epsilon^{-\frac{3 T}{r c}}
\end{aligned}
$$

amperes 
In the steady state there is a current in branch III of value,

$$
\frac{E}{3 r}
$$

amperes

Adding to this the negatives of the two discharge currents above gives the current in branch III due to the sudden application of voltage,

$$
i_{\mathrm{II}}=\frac{E}{3 r}-\frac{E}{2 r} \epsilon^{-\frac{t}{r c}}+\frac{E}{6 r} \epsilon^{-\frac{3 t}{r c}}
$$

amperes

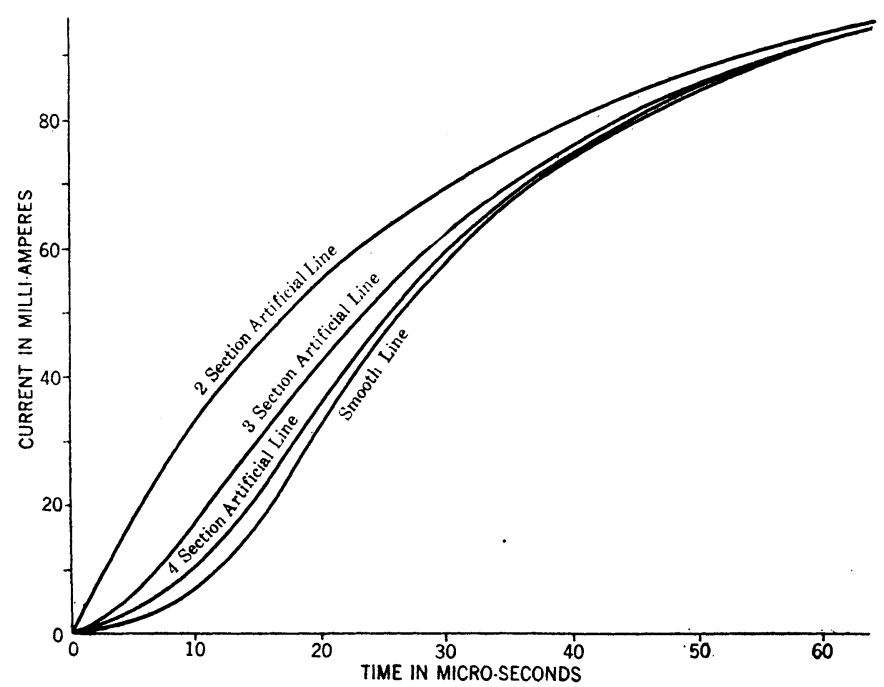

Fig. 7-Received Current on Grounded Transmission LineSmooth and Lumped Lines

Suddenly applied steady voltage at home end-20 volts

Line resistance $200 \mathrm{ohms}$ - line capacity $10^{-6}$ farads

or in terms of total constants,

$$
i_{\mathrm{III}}=\frac{E}{R}-\frac{3 E}{2 R} \epsilon^{-\frac{9 t}{R c}}+\frac{E}{2 R} \epsilon^{-\frac{27 t}{R c}}
$$

amperes

A part of this example is treated more fully in the appendix.

A series of curves of this type, together with the smooth line curve from Kelvin's formula, are plotted in Fig. 7 for typical constants.

\section{APPENDIX}

\section{Algebra of Example III}

In the steady state condenser I (Fig. 5) is charged to potential $\frac{2 E}{3}$. The discharge of this condenser is computed as follows: 
Consider branch I the main branch, and form the generalized impedance of the network by combining the generalized impedance of the elements in the manner of simple resistances.

$$
Z_{\mathrm{I}}=\frac{1}{c n}+\frac{1}{\frac{1}{r}+\frac{1}{r+\frac{1}{c n+\frac{1}{r}}}}
$$

ohms

or reducing,

$$
Z_{\mathrm{I}}=\frac{r^{2} c^{2} n^{2}+4 r c n+3}{c n(2 r c n+3)}
$$

ohms

Equate to zero, solve for $n$, and we obtain as free generalized angular velocities,

$$
\begin{aligned}
& n_{1}=-\frac{1}{r c} \\
& n_{1}=-\frac{3}{r c}
\end{aligned}
$$

hyp. rad./sec.

Differentiate $Z_{\mathrm{I}}$ with respect to $n$, multiply by $n$, and we obtain the threshold impedance. It is well to use for this purpose the form of $Z_{\mathbf{I}}$ which is most easily differentiated.

$$
T_{\mathrm{I}}=n \frac{d Z_{\mathrm{I}}}{d n}=-\frac{1}{c n}-\frac{r^{2}}{(2 r c n} \quad \text { ohms. }
$$

This threshold impedance has two values corresponding to the two free generalized angular velocities obtained above. Substituting them in and reducing, there results,

$$
\begin{aligned}
T_{1} & =2 r \\
T_{2} & =\frac{2 r}{3}
\end{aligned}
$$

ohms

Divide the initial voltage, $\frac{2 E}{3}$, by each of these values of the threshold impedance to give the amplitudes of oscillation of the discharge current:

$$
\begin{aligned}
& A_{1}=\frac{E}{3 r} \\
& A_{2}=\frac{E}{r}
\end{aligned}
$$


Associate these amplitudes with the corresponding generalized angular velocities, and we have the discharge current of condenser I:

$$
i_{\mathrm{I}}=\frac{E}{3 r} \epsilon^{-\frac{t}{r c}}+\frac{E}{r} \epsilon^{-\frac{3 t}{r c}}
$$

amperes.

This current divides among the several branches in proportion to their generalized admittances. The proportional part which passes through branches II and III is thus,

$$
\frac{r}{2 r+\frac{1}{c n+\frac{1}{r}}}
$$

numeric

and of this the part

$$
\frac{\frac{1}{c n}}{r+\frac{1}{c n}}
$$

numeric

goes through branch III. The proportional part in branch III is thus,

$$
\frac{r}{2 r+\frac{1}{c n+\frac{1}{r}}} \times \frac{\frac{1}{c n}}{r+\frac{1}{c n}}
$$

numeric

or

$$
\frac{1}{2 r c n+3}
$$

numeric.

This ratio again has two values corresponding to the two free values of $n$. Substituting these we obtain for the ratio the two values,

$$
1 \text { and }-\frac{1}{3}
$$

To multiply $i_{\mathrm{I}}$ by the ratio, we multiply each term by the value of the ratio which corresponds to the value of $n$ already involved 
in the term. In this manner we obtain the portion of the discharge current of condenser I which passes through branch III:

$$
i_{\mathrm{III}}^{\prime}=\frac{E}{3 r} \epsilon^{-\frac{t}{r^{c}}}-\frac{E}{3 r} \epsilon^{-\frac{3 t}{r_{i}}}
$$

amperes.

The discharge of condenser II is treated in like manner.

In this example the generalized angular velocities contain no imaginary part. In the more general case with inductance present, the generalized angular velocities are complex. The method of procedure is identical in the two cases; and the only difficulty results from the length of the expressions involved.

List of Symbols Employed

$A$ = Amplitude of sinusoidally varying quantity.

$j=$ The pure imaginary $\sqrt{-1}$.

$\epsilon \quad=$ Naperian base. $2.718 \ldots$

$\omega=$ Angular velocity. Radians per second.

$t=$ Time in seconds.

$n=$ Generalized angular velocity. Hyperbolic radians per second. $\angle$

$\alpha=$ Logarithmic decrement. Hyperbolic radians per second.

$C, c=$ Capacitance. Farads.

$L=$ Inductance. Henries.

$R, r=$ Resistance. Ohms.

$Z$ = Generalized impedance. Ohms. $\angle$

$T=$ Threshold impedance. Ohms. $\angle$

$E=$ Initial potential. Volts.

$\dot{e}=$ Instantaneous potential. Volts.

$i=$ Instantaneous current. Amperes

$\angle \quad=$ Sign used to denote a complex quantity. 
Discussion on "Oscillating-Current Circuits by the Methods of Generalized Angular Velocities" (Bush), New York, February 15, 1917.

A. E. Kennelly: Mr. Bush's paper points out a simple method of making the oscillating-current circuit as easily managed by engineers as the alternating-current circuit. The only essential difference is that the coefficient with which you multiply the inductance, or multiply the capacitance of a circuit, instead of being a pure $j$ quantity, or a pure imaginary, is a complex quantity, which contains a real component.

The important and new material which Dr. Bush presents here is the threshold impedance. By the use of this conception, you are enabled to obtain the initial strength of the oscillating current in the circuit at a time when the impedance has to be made zero. If you make the impedance of a circuit zero you are unable, ordinarily, to determine what the initial current strength of discharge will be; but by taking this threshold value of the impedance just at the time when it is about to become zero (the disappearing value, so to speak), you are able in the manner in which he shows here to determine the starting-off current, and then the natural decay, using the real part of the complex angular velocity, to determine the strength of the current at all subsequent times. I think as soon as this method is clearly understood by engineers, they will be able to use it with great effectiveness, because it destroys, as the author has said, the necessity of introducing differential equations every time we have an oscillating-current system to deal with. In the early days of the a-c. circuit, we all had to start off with differential equations, and get the solutions of an alternating current in that manner. Since then, we have found it unnecessary to go to differential equations, we have reserved that for final work in physics, and we can work with a generalized Ohm's law. Now, here is a second generalization and extension of Ohm's law, whereby, without any reference to differential equations, we get right down to Ohm's-law equations of an oscillatingcurrent circuit and obtain solutions by the ordinary method of vector arithmetic.

C. Fortescue: It appears to me that there is not much advantage in this method of considering the free oscillations of a network, if the order of the differential equations for the problem under consideration is beyond the third order. In fact, the method is really based on the use of differential equations. The generalized angular velocity is simply an equivalent for differential operator $D$, and I think sometimes that we lose something in adopting a narrower method of dealing with these problems than that of differential equations. It seems to me that on a new problem, if one were not able to form the differential equations of the system some difficulty would be found in using this method.

H. Fletcher: I would like to inquire whether the method can be used in cases where the e.m.f. was different from the steady e.m.f. 
Thornton C. Fry: I want to call attention in the first place to the fact that the chief element of interest in this method lies in the fact that it brings in the natural periods of the circuit on the application of the e.m.f. I don't think there are many people who are interested in a-c. problems who have not known that the use of the generalized impedance formula, with $j \omega$, was also possible where the alternating e.m.f. was damped, and consequently $d t$ must be replaced by a complex, as distinguished from a pure imaginary quantity.

The second point that I would like to make is the fact that while Mr. Bush has given a proof of Heaviside's formula from the point of his Fourier integral on, he did something just at the time of introducing his Fourier integral which is not justified mathematically. He introduced an operator in the integral sine. That is equivalent to a differentiation in the sine of integration, or an integration in the sine of integration, or more generally a combination of the two; and at the same time, in having thrown mathematical rigor to the winds at that point, he would have been justified in going ahead and throwing mathematical rigor to the winds further, and carrying out his solution by purely operational methods. It is much simpler, and undoubtedly Mr. Bush has done that for himself. The method simply consists in noting that the rate is itself a fraction introducing $D$ only to integral powers, and can consequently be expanded into a series of partial fractions. Everybody knows how to do that, but everybody does not know Lorentz' series; and then by the theory of Lamique, with which the author seems to be familiar, he can perfectly well integrate each one of those partial fractions and come to the result that he has. This is a thing which is more useful for the practical man, because he can expand a long series of partial fractions, but he cannot know Lorentz' series. Of course, Mr. Bush can do that by introducing his arbitrary e.m.f. as distinguished from his absolutely constant e.m.f., but in each case he would have to carry out his line of reasoning to evaluate the residue, this function at the various variants in order to be able to write down his Lorentz series, which is entirely unnecessary by the other method.

V. Bush: On the first point, made by Mr. Fortescue, perhaps I can give an idea of the amount of simplification which results from the use of this method by quoting one problem. Take the case of an inductively-coupled circuit, which I left out of the paper, because I believe it is of sufficient importance to warrant special treatment. In that case we encounter the same fourth degree equation, whether we solve by differential equations or by threshold impedance. But in the determination of the constants of integration, in the differential equation method, we encounter fourth order determinants with complex members; whereas in the case of using the threshold impedance it is merely a question of substituting our complex values of the generalized angular velocity into a simple algebraic expression. The work 
for a typical circuit I believe will be much more than cut in half for a complete solution.

Now, in regard to whether this method can be used for the study of the application of other voltages than the steady voltage: Heaviside, I believe, does this in a few special cases. I have used the method and obtained correct results in the study of the sudden application of an alternating e.m.f. to certain simple networks, but the method there rapidly becomes involved, so that there is no great advantage.

Concerning the rigor of Wagner's proof of the Heaviside formula: I shall be glad to study this proof again. My purpose in presenting it here was rather to show the limitations of the method than to rigorously establish it.

In regard to the expansion of the operator into partial fractions: I think you will find that for circuits with lumped constants the threshold impedance method will be shorter. For circuits with distributed constants, to which the method can also be applied, an expansion in partial fractions I have found to be valuable; in fact, I have found it to be superior to Heaviside's method of expansion in power series; but for the ordinary network with lumped constants which we meet in practise, I think you will find the threshold impedance method superior, the difficulties being simply algebraic ones, with the greater part of the difficult mathematical work left out.

A. Press (Communicated after adjournment): Mr. Bush has done the electrical profession a very great service in drawing attention to the Heaviside Symbolic Method of dealing with Transient, i.e., Impact. Phenomena. The paper on "Oscillating Currents by the Method of Generalized Angular Velocities" is particularly well timed, especially since the "Shock Problems" are forcing consideration with their Transient Oscillating Currents, whether in interconnected power circuits or in heavy electrical machinery. However, it is highly regrettable that $\mathrm{Mr}$. Bush did not give a more complete theoretical development of the Heaviside operator methods used, but rather assumed that his readers were, or could easily become, acquainted with them.

A more detailed exposition would have brought out for example that Kirchoff's laws are being interpreted in two totally different ways, which can lead to error if care is not taken. In the case of networks it is very important to state that Kirchoff's laws really apply to instantaneous values and it is only by means of peculiar extensions that a so-called "Ohms Law" very much similar to the direct-current law can be employed in practical problems.

The basis of all such symbolic work is really the set of simultaneous differential equations linking up the current in the network, and from which the resolved expressions of current are to be derived algebraically to obtain the threshold impedance factors. Another matter dealt with is the proper expression for what Mr. Bush calls the "Generalized Velocities." 
Undoubtedly Mr. Bush's method will give the independent frequency terms but to obtain the amplitude factors it is still necessary to fall back on the fundamental differential equations of the net-work. The paper is replete with suggestions, yet, because the import of Heaviside methods are so little understood the writer takes the liberty of dilating on Mr. Bush's work and incidentally gives a simple and original proof of Heaviside's famous "Expansion Theorem".

The Basis for Vectorial Representation. For a simple circuit with resistance and self induction the differential equation is

$$
e=i R+L \frac{d i}{d t}
$$

if then

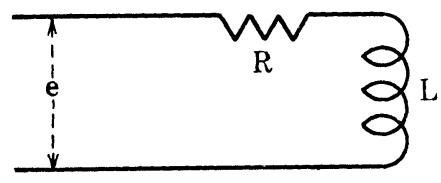

$$
\begin{aligned}
& i=I \sin \omega t, \text { then substituting, } \\
& e=I R \sin \omega t+L \omega I \cos \omega t
\end{aligned}
$$

These are all instantaneous values and therefore equation (2) suggests a method of representing the relation of the two variables $e$ and $i$.

If the amplitudes $I$ and $E$ are to be taken, then geometrically one can represent the state of affairs indicated by equation (1)

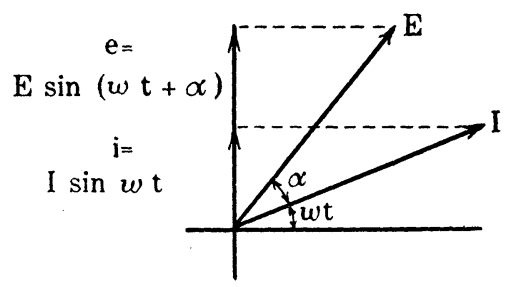

as follows: Where $\alpha$ is the angle of lag between $E$ and $I$ and is a constant of the circuit depending in fact upon the constants occurring in the differential equation (1). The g e o metric representation therefore is a complete solution of the steady case.

Projecting the lines $E$ and $I$ upon the axis at right angles to the one from which $\omega t$ is reckoned one has

$$
\begin{aligned}
& e=E \sin (\omega t+\alpha) \\
& i=I \sin \omega t
\end{aligned}
$$

and therefore it is required to show that

$$
e=E \sin (\omega t+\alpha)=I R \sin \omega t+L \omega I \cos \omega t
$$

by equation (2). Developing $\sin (w t+\alpha)$ one has by ordinary trigonometry

$e=E \sin \omega t \cos \alpha+E \cos \omega t \sin \alpha=I R \sin \omega t+L a I \cos \omega t$ 
If then (4) is true it will be true for $\omega t=0$ and for $\omega t=\pi / 2$. Thus for $\omega t=\pi / 2$ one has

$$
E \cos \alpha=I R
$$

Again for $\omega t=0$ one has.

$$
E \sin \alpha=L I \omega
$$

and dividing (6) by (5) one obtains

$$
\sin \alpha \cos \alpha=\tan \alpha=L \omega / R \text {. }
$$

Basis for Symbolic and Exponential Representations. However, because we are dealing with sinusoids we have the following:

$$
\begin{aligned}
& \frac{d}{d t} \cdot \sin \omega t=\omega \cdot \cos \omega t \\
& \left(\frac{d}{d t}\right)^{2} \cdot \sin \omega t=-\omega^{2} \cdot \sin \omega t \\
& \left(\frac{d}{d t}\right)^{3} \cdot \sin \omega t=-\omega^{3} \cdot \cos \omega t \\
& \left(\frac{d}{d t}\right)^{4} \cdot \sin \omega t=\omega^{4} \cdot \sin \omega t, \text { etc. }
\end{aligned}
$$

so that for $d / d t$ one can actually replace $w j$, and where for any even power of $j$ one is entitled to write $j^{2}=(\sqrt{-1})^{2}=-1$, but for odd powers one must fall back upon differentiation when necessary.

Thus $j^{3}=j^{2} \cdot j=-1 j=-\frac{1}{\omega} \cdot \frac{d}{d t}$, because equivalently

$$
\frac{d}{d t}=\omega i
$$

Equation (1) above can therefore be written algebraically

$$
e=(R+L \omega j) i
$$

and from the geometric construction one can write (7) vectorially by capitalization and dotting as

$$
E=(R+L \omega j) !
$$

so that $E$ and $I$ are now vectors in the amplitude time-phase plane. The multipliers $j$ here must mean that a line $L \omega$ is to 
be drawn perpendicular to $R$ which latter is to be drawn in the direction of $I$ and thereby one obtains the angle $\alpha$ and the direction of $\dot{E}$.

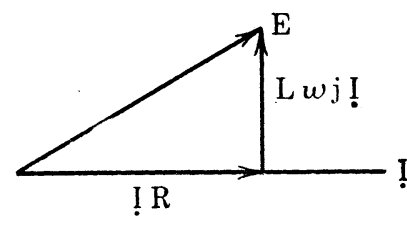

The quantity $i$ therefore is an indicator how to draw the quantity $L \omega I$, for $L \omega j I$ is perpendicular to the quantity $I$.

There is however, an exponential method of representation, for since one has the equation (7)

$$
e=(R+L \omega j) i,
$$

and by ordinary algebra

$$
\begin{aligned}
\epsilon^{\omega t} & =1+\frac{\omega t}{11}+\frac{\omega^{2} t^{2}}{12}+\frac{\omega^{3} t^{3}}{13}+ \\
\cos \omega t & =1-\frac{\omega^{2} t^{2}}{12}+\frac{\omega^{4} t^{4}}{14}-\frac{\omega^{6} t^{6}}{16}+ \\
\sin \omega t & =\omega t-\frac{\omega^{3} t^{3}}{13}+\frac{\omega^{5} t^{5}}{15}-
\end{aligned}
$$

and it follows that

$$
A e^{j \omega t}=A(\cos \omega t+j \sin \omega t) .
$$

The utility of this mode of representation is important.

Any vector such as $E$ or $I$ in a time-phase diagram can always be considered as made up of two components at right angles to each other. Thus choosing any datum line for $\omega t$ one can write

$$
I=I(\cos \omega t+j \sin \omega t) \text {. }
$$

Thus when one writes $A e^{j \omega t}$ it really means

$$
A(\cos \omega t+j \sin \omega t)=A=A e^{j \omega t}
$$

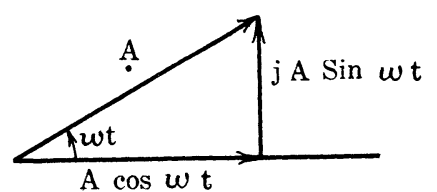

the same magnitude but only varies its position in the plane.

The current vector $I$ before mentioned could be written therefore

$$
I=I e^{j \omega t}
$$

Such a wave of current would be represented by a true sine wave. 
Bush's Generalized Exponential Representation. Supposing, however, that we wish to represent a decremental wave component of the following form:

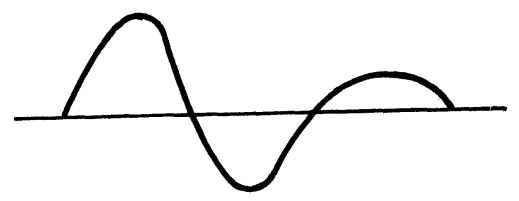

such as one has in wireless or as one has in some direct-current circuits when the switch is suddenly closed or opened and there is considerable capacity and self-induction. A wave of this type is represented by

$$
\begin{aligned}
& i=I e^{-a t} \sin \omega t, \text { or } \\
& i=I e^{-a t} \cos \omega t
\end{aligned}
$$

Here counting $\omega t$ as before the projection of the constant amplitude vector $I$ upon the projection axis would not give the true instantaneous value because as $t$ continually grows in $\omega t$ it is necessary also to always consider the amplitude multiplying factor $e^{-a t}$. This can be corrected for by applying the factor $e^{-a t}$ to each position of the original amplitude vector $I_{c}$ as in fact this original amplitude vector travels around.

As a true vectorial representation one can therefore write

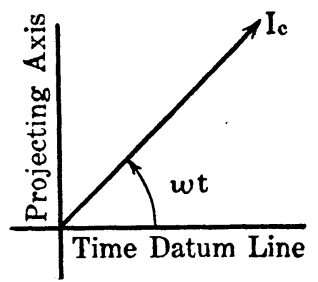

$$
\begin{gathered}
I=e^{-a t} I_{c}(\cos \omega t+j \sin \omega t)=I_{c} e^{-a t} e^{j t \omega} \\
=I_{c} e^{(-a+j \omega) t}=I_{c} e^{n t},
\end{gathered}
$$

where $n$ is a complex and is of the form

$$
n=-\alpha+j \omega .
$$

In the diagram just given $\omega t$ was drawn as a real angle. Supposing however, that it is assumed that in some imaginary geometric system complex angles of the form

$$
\frac{n}{j}=-\frac{\alpha}{j}+\omega=j \alpha+\omega
$$

could be drawn then as the vector $I_{c}$ travelled around according to the angular velocity $j \alpha+\omega$, projecting on to the projecting axis would actually give the time instantaneous values of $i$.

The complex angular velocity should strictly speaking be given by dividing the complex exponent in $e^{j \omega t}$ by $j t$ and the 
same thing should hold for $e^{n t}$. This view point as to complex angles is not Heavisidean but is really due to Mr. Bush in his paper except for the fact that a division by $j t$ is not resorted to. Thus if $\alpha=0$, the amplitude factor is $e^{-a t}=1$ then,

$$
n=j \omega
$$

and it was seen that $\omega$ was called the angular velocity and not $j \omega$.

Application of Generalized Velocity Components to Circuits. Now take the equation of a circuit in which there is capacity,

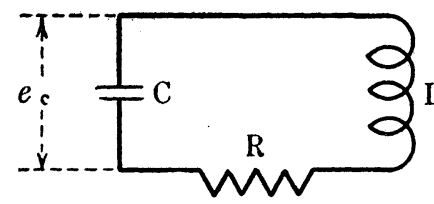
inductance and resistance, if $i$ is the current then we have as the voltage across the resistance, $i R$ : $L$ for that across the inductance $L \frac{d i}{d t}=n L i$ writing $n$, for $d / d t$

but for the capacity the voltage is not so straight forward, if we wish to express the voltage in terms of $i$ and $C$. We have

$$
q=C e_{c}
$$

where $q$ is the charge and $C$ is the capacity in farads for an impressed e.m.f. $e_{c}$, so that

$$
\begin{gathered}
\frac{d q}{d t}=i=\frac{d e_{c}}{d t}=n C e_{c}, \text { since } n=\frac{d}{d t}, \text { whence } \\
e_{c}=\frac{i}{C n}
\end{gathered}
$$

The equation of a circuit in which the impressed voltage is zero and forms the right hand term is therefore

$$
\left(R+L n+\frac{1}{C n}\right) i=0
$$

Heaviside's Treatment of Zero Operands. An equation of this type is to be solved in the following manner by Heaviside. If both sides of the equation are operated upon with what really is a differential operator function, $\left(R+n L+\frac{1}{C n}\right)^{-1}$ whatever this may mean, will give

$$
\begin{aligned}
\left(R+n L+\frac{1}{C n}\right)^{-1}(R+n L+ & \left.\frac{1}{C n}\right) \cdot i \\
& =\left(R+n L+\frac{1}{C n}\right)^{-1} \cdot 0
\end{aligned}
$$


The left-hand side gives the $i$ because any quantity (or operation) in ordinary algebra when multiplied by its reciprocal is assumed to give unity and therefore one has

$$
i=\frac{0}{R+n L+\frac{1}{C n}}
$$

where it must now be understood that zero is the operand and corresponds to the impressed disrupted voltage.*

"Determinantal Equation". Resolution into Partial Fractions. Supposing, however, the right-hand side is developed in partial fractions then algebraically it will be necessary to put

$$
R+n L+\frac{1}{C n}=0
$$

This equation of condition is called by Heaviside the "Determinnantal Equation." $\dagger$

As an equation in $n$ we will have

$$
n=-\frac{R}{2 L} \pm j \sqrt{\frac{1}{L C}-\left(\frac{R}{2 L}\right)^{2}}=-\alpha \pm j \omega
$$

so that by the rule for partial fractions one can write (11) as

$$
i=i_{1}+i_{2}=\frac{0}{n+(\alpha-j \omega)}+\frac{0}{n+(\alpha+j \omega)}
$$

But then how interpret the individual fractions?

Let us take a single term given by

$$
\begin{gathered}
i_{1}=\frac{0}{n+(\alpha-j \omega)} \text { or, }\{n+(\alpha-j \omega)\} i_{1}=0 \\
\frac{d i_{1}}{d t}+(\alpha-j \omega) i_{1}=0 .
\end{gathered}
$$

The solution of this last is very well known and gives

$$
i_{1}=A e^{-(a-j \omega) t}
$$

\footnotetext{
*For fractions of this type compare Perry's Calculus, page 238.

† See also Bush's paper page 209 .

†See Perry's Calculus, page 238.

\$One must be very careful not to treat the numerator zero, in such an operator function as still zero when multiplied by any function. To do so will result in wrong amplitude factors or determinantel equations.
} 
o that the complete solution of the above is really

$$
\begin{aligned}
i & =\left\{e^{-(a-j \omega) t}+e^{-(a+j \omega) t}\right\} A=A e^{-\alpha t}\left(e^{j \omega t}+e^{-j \omega t}\right) \S \\
& =A e^{-\alpha t} \cos \omega t
\end{aligned}
$$

Turning now to what has been called the "Generalized Angular Velocity" it is in fact nothing other than what constitutes the roots of the Determinantal Equation in $n$ considered as an ordinary algebraic equation and divided by $j$. This is the complex "Generalized Velocity" of Bush. However, $n$ itself is not a generalized velocity but rather a Heaviside-Operator function. The actual velocity assuming the resistance to be sufficiently small is given by,

$$
\begin{aligned}
\frac{j \omega}{j} & =\sqrt{\frac{1}{L C}-\left(\frac{R}{2 L}\right)^{2}}=\omega \\
f & =\frac{1}{2 \pi} \sqrt{\frac{1}{L C}-\left(\frac{R}{2 L}\right)^{2}}
\end{aligned}
$$

where $f$ is the frequency of the oscillations.

Heaviside's Expansion Theorem. Engineers encounter linear differential equations when dealing with the "Forced Vibrations" of a steadily applied alternating force and "Natural Vibrations" set up by the shock application of some force. The latter call for evanescent terms which mathematically correspond to the "complementary function" whereas for that part corresponding to the steady state particular solutions are found necessary.*

Heaviside's Expansion Theorem is directed toward determining the evanescent terms in a mechanical or electrical dynamic system, without having to laboriously evaluate constants as required by the usual methods of solutiont Moreover it allows of semi-graphic methods of obtaining the amplitude coefficients in complicated and transcendental expressions. It is especially important in net works $\ddagger$ where make and break contacts are made. Yet on closer examination the whole complicated phenomenon can be looked upon as being due to a series of transient terms ' $\mathrm{X}$ ' having their origin in the steepness of the voltage curve at the make and break (really the complementary solution in mathematics) plus a term due to the steady variable (or non-variable) state corresponding to the forced vibration. The latter is the so-called particular solution.

§See Perry, page 520.

*See Differential Equations by Abraham Cohen, page 91.

†See Heaviside, E. M. T. Vol. II, pages 127-129.

†Alternate-current networks should be solved by means of Kirchoff's Laws vide Fleming. Alternate Current Transformer, Vol. I, page 234. 
For the general case with the suddenly applied voltage $E_{0}$ impressed applied to a circuit of which the differential equation of current is

$$
F(\theta) . i=E_{0} \dagger
$$

the steady state will be given by placing $\theta=0$ in $F(\theta)$ so that

$$
i_{\text {steady }}=\frac{E_{0}}{Z_{0}}
$$

where $Z_{0}$ means $F(\theta)$ in which $\theta$ is put equal to zero. To find the transient component $X$ due to shock one has by (13) and (14)

$$
X=\frac{E_{0}}{Z}-\frac{E_{0}}{Z_{0}}=\frac{Z_{0}-Z}{Z_{0} Z} \cdot E_{0}
$$

In general all the terms in $Z_{0}-Z$ will contain $\theta$ whence dividing by $\theta$ one can write

$$
X=\frac{\left(Z_{0}-Z\right) /\left(\theta Z_{0}\right)}{Z} \cdot \theta E_{0}
$$

Now splitting up $\frac{Z_{0}-Z}{\theta Z_{0} Z}$ into partial fractions of the form $\sum \frac{A_{n}}{\theta+a_{n}}$, let $\varphi(\theta)=\frac{Z_{0}-Z}{\theta Z_{0}} ; F(\theta)=Z$ then

$$
A_{n}=\frac{\varphi(\theta)}{\left\{\frac{d}{d \theta} \cdot F(\theta)\right\}_{\theta=\theta_{n}}}
$$

where $\theta_{n}$ is the $n$th root of the determinantal equation $F(\theta)=0$ regarded as an ordinary algebraic equation in $\theta$. Since

$$
\varphi(\theta)=\frac{Z_{0}-F(\theta)}{\theta Z_{0}}
$$

then replacing $\theta$ by one of the roots it follows that $F(\theta)=0$ and

therefore $\varphi(\theta)_{n}=\frac{1}{\theta_{n}}, A_{n}=\frac{1}{\left\{\theta \frac{d Z}{d \theta}\right\}_{\theta=\theta_{n}}}$

*Todhunter's Integral Calculus, 7 th edition, page 26 .

$\dagger$ The symbol $\theta$ is used for $d / d t$. 


$$
X=\sum \frac{1}{\left(\theta \frac{d Z}{d \theta}\right)_{\theta=n_{n}}} \cdot \frac{\theta E_{0}}{\theta-\theta_{n}}
$$

As already pointed out

$$
\theta E_{0} /\left(\theta-\theta_{n}\right)=E_{0} \cdot \frac{0}{\theta-\theta_{n}}=E_{0} e^{\theta} n^{t}
$$

and therefore finally, for the transient component with suddenly applied voltage

$$
X=E_{0} \sum\left\{\frac{e^{\theta t}}{\theta \frac{d Z}{d \theta}}\right\}_{\theta=\theta_{n}}
$$

which expresses Heaviside's Expansion Theorem.

Application. A direct-current voltage is suddenly impressed upon a series circuit containing inductance resistance and capac-

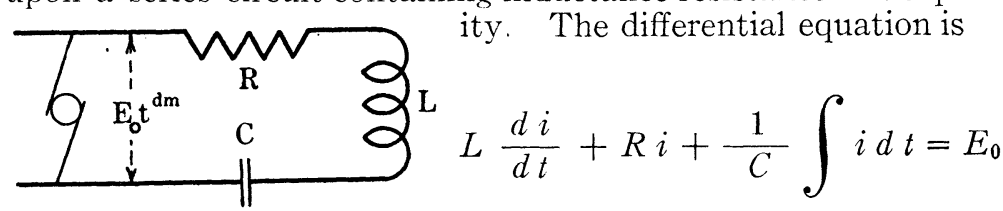

or

$$
i=\frac{E_{0}}{L \theta+R+\frac{1}{C \theta}}=\frac{\text { Voltage }}{\text { Impedance Operator }}
$$

Thus for the impedance operator one has

$$
Z=L \theta+R+\frac{1}{C \theta}
$$

and regarding momentarily $Z=0$ as an algebraic equation in $\theta$ the roots are

$$
\begin{aligned}
& \dot{\theta_{1}}=-\frac{R}{2 L}+\sqrt{\frac{R^{2}}{4 L^{2}}-\frac{1}{L C}} \\
& \theta_{2}=-\frac{R}{2 L}-\sqrt{\frac{R^{2}}{4 L^{2}}-\frac{1}{L C}}
\end{aligned}
$$


Thus $\theta \frac{d Z}{d \theta}=L \theta-\frac{1}{C \theta}$ and therefore for the transient current

$$
i_{x}=\frac{E_{0} \cdot e^{\theta_{1} t}}{L \theta_{1}-\frac{1}{C \theta_{1}}}+\frac{E_{0} e^{\theta_{2} t}}{L \theta_{2}-\frac{1}{C \theta_{2}}}
$$

This can be put in another form, since in developing the same, use was made of the equation $Z=0$ in determining the roots. Thus

$$
\begin{aligned}
& L \theta_{1}+R=-\frac{1}{C \theta_{1}} ; \quad L \theta_{2}+R=-\frac{1}{C \theta_{2}} \text { whence } \\
& i_{x}=\frac{E_{0}}{2 L \theta_{1}+R} e^{\theta_{1} t}+\frac{E_{0}}{2 L \theta_{2}+R} e^{\theta_{2} l} .
\end{aligned}
$$

